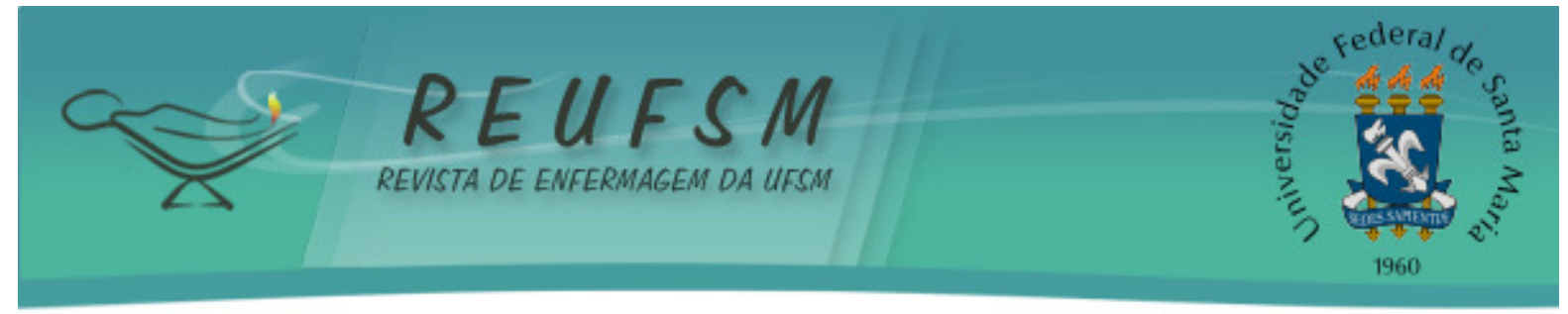

ARTIGO ORIGINAL

\title{
A PRESCRIÇÃO DE MEDICAMENTOS NA ESTRATÉGIA DE SAÚDE DA FAMÍLIA: PERCEPÇÃO DE ENFERMEIROS
}

\section{PRESCRIPTION OF MEDICINES IN FAMILY HEALTH STRATEGY: NURSES' PERCEPTION PRESCRIPCIÓN DE MEDICAMENTOS EN LA ESTRATEGIA DE SALUD FAMILIAR: PERCEPCIÓN DE LAS ENFERMERAS}

Maria Neyrian de Fátima Fernandes ${ }^{1}$ Keith Suelen de Moura Lopes ${ }^{2}$ Doi: $10.5902 / 2179769220713$

Fabrício Augusto Chaves Rocha ${ }^{3}$

RESUMO: Objetivo: conhecer a percepção dos enfermeiros sobre a prescrição de medicamentos, realizada pela categoria na Estratégia de Saúde da Família de um município no Maranhão. Método: estudo de abordagem qualitativa, descritivo, tendo como participantes oito enfermeiras. Dados coletados em abril de 2014 por meio de entrevistas e analisados de acordo com o método de análise de conteúdo. Resultados: constatou-se que as enfermeiras entrevistadas são jovens, recém-admitidas no cargo, com tempo de formação superior a três anos. A partir dos discursos, formularam-se duas categorias: percepção dos enfermeiros sobre a sua prescrição de medicamentos e as bases do conhecimento para a prescrição. Detectou-se que as enfermeiras veem a prescrição de medicamentos como uma atribuição importante e necessária, sentindo-se preparadas para prescrever devido a sua vivência profissional e não a sua formação acadêmica. Considerações Finais: aponta-se a necessidade de que os cursos de graduação preparem devidamente os enfermeiros para desempenhar essa função.

Descritores: Enfermagem; Estratégia saúde da família; Prescrições de medicamentos.

ABSTRACT: Aim: to know nurses 'perception about medicine prescription in Family Health Strategy in a city in the State of Maranhão. Method: qualitative and descriptive study, in which eight Nurses were interviewed. Data were collected in April 2014 through interviews and analyzed according to the content analysis method. Results: it was noted that the interviewed nurses were recently hired young women that had been graduated for more than three years. From the interviews, two categories emerged: nurses' perception on their medicine prescribing and knowledge bases for prescription. It was perceived that nurses see prescription of drugs as an important and necessary role, they argued that the knowledge used to prescribe is from their professional experience rather than their academic formation. Final considerations: thus, the need of undergraduate courses to properly prepare nurses to perform such function is highlighted.

Descriptors: Nursing; Family health strategy; Drugs prescriptions.

RESUMEN: Objetivo: conocer la percepción de las enfermeras sobre su prescripción de medicamentos en la Estrategia de Salud Familiar de una ciudad del Maranhão. Método:

\footnotetext{
1 Enfermeira. Professora Assistente II da Universidade Federal do Maranhão/UFMA, Imperatriz, Maranhão, Brasil. Doutoranda do Programa Enfermagem Psiquiátrica da Escola de Enfermagem de Ribeirão Preto da Universidade de São Paulo, Ribeirão Preto, São Paulo, Brasil. E-mail: neyrianfernandes@gmail.com

2 Enfermeira pela Universidade Federal do Maranhão/UFMA, Imperatriz, Maranhão, Brasil. E-mail: keith.luz@hotmail.com

3 Enfermeiro pela Universidade Federal do Maranhão/UFMA, Imperatriz, Maranhão, Brasil. E-mail: fabriciorocha.ac@gmail.
} 


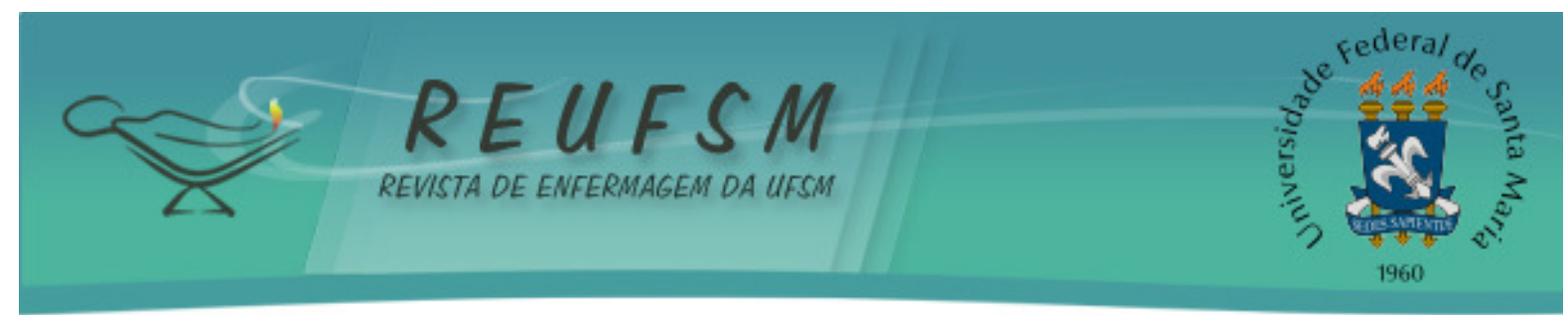

estudio descriptivo, cualitativo, con ocho enfermeras participantes. Los datos fueron recogidos en abril de 2014 a través de entrevistas y analizados según el método de análisis de contenido. Resultados: se observó que las enfermeras entrevistadas son mujeres jóvenes, recién admitidas en oficina y tienen más de tres años de entrenamiento. A partir de los discursos, se encontró dos categorías: la percepción de las enfermeras sobre sus prescripciónes de medicamentos y las bases de conocimiento para la prescripción. Se detectó que las enfermeras ven medicamentos recetados como algo importante y necesario, y siéntense dispuestas a recetar debido a su experiencia profesional y no su formación académica. Consideraciones finales: el estudio señala la necesidad de cursos que preparen adecuadamente la enfermera para realizar esta función.

Descriptores: Enfermería;Estrategia salud familiar; Prescripciones de medicamentos.

\section{INTRODUÇÃO}

O profissional enfermeiro exerce um papel decisivo e proativo na promoção e prevenção da saúde dos indivíduos em suas diversas dimensões, assim como na identificação das necessidades de cuidado da população. ${ }^{1}$

No contexto da Estratégia Saúde da Família (ESF), modelo que visa à reorganização da Atenção Primária à Saúde (APS) no país, seguindo os preceitos constitucionais do Sistema Único de Saúde (SUS) como a integralidade, o enfermeiro exerce os papéis de atenção à saúde e de gestão na atenção básica. Esses papéis contribuem para a ampliação da resolubilidade de problemas, exercem impacto na qualidade de vida dos indivíduos, e das coletividades e proporcionam importante relação custo-efetividade. ${ }^{2}$

Assim, na ESF, o enfermeiro atende à comunidade e desenvolve atividades de promoção, educação e prevenção à saúde, assim como o tratamento e reabilitação dos usuários. Tais atribuições têm proporcionado autonomia no exercício profissional do enfermeiro na APS, resultando em uma significante ascensão social e política da profissão, contribuindo para avanços no saber e fazer da enfermagem. ${ }^{2-3}$

$\mathrm{Na}$ APS, o enfermeiro executa o ato de prescrever e transcrever medicações conforme protocolos estabelecidos nos programas do Ministério da Saúde (MS) e disposições legais da profissão. Programas propostos pela APS, como o Plano de Reorganização da Atenção à Hipertensão Arterial e ao Diabetes Mellitus, o Programa Nacional de Controle da Tuberculose (PNCT) e o Programa Nacional de Controle da Hanseníase, viabilizam que o enfermeiro atue a partir dos primeiros problemas relatados pelo usuário. Desta maneira, é possível investigar a situação por meio do levantamento de dados concretos para os possíveis diagnósticos compatíveis com o arcabouço legal da profissão, intervenções e, finalmente, uma possível prescrição. ${ }^{4-5}$ Esses procedimentos proporcionam, então, que o usuário receba o cuidado de um único profissional, com efetividade e em um tempo menor.

Dessa forma, pode-se considerar a prescrição/transcrição medicamentosa um avanço, porque confere autonomia ao enfermeiro, assim como the possibilita prover um cuidado sistêmico ao paciente. Nesse contexto, o cuidado de enfermagem pode ser bem aceito pelo usuário, promovendo melhoria no acesso ao tratamento e elevando a sua satisfação. ${ }^{5}$

As atividades desenvolvidas por enfermeiros na APS representam uma mudança no paradigma da atenção e dos cuidados em saúde, outorgando a esse profissional um papel de destaque nas equipes multidisciplinares. ${ }^{6}$

Além do Brasil, países como Austrália, Canadá, França, Reino Unido, EUA, Suécia, Botswana, Zâmbia, Nova Zelândia, Holanda, África do Sul e Irlanda já regulamentaram a prescrição de medicamentos pela enfermagem. ${ }^{7-8}$ Estender o direito de prescrição para 0 


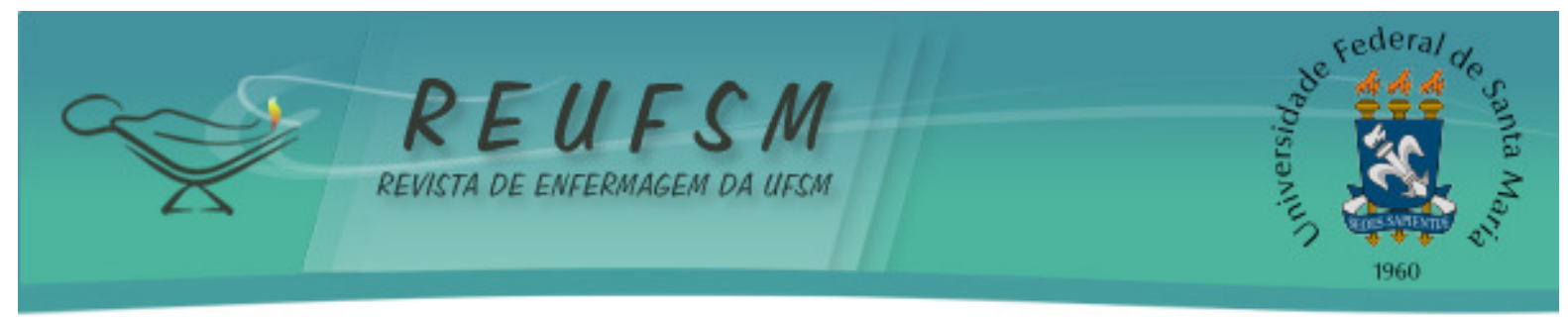

enfermeiro possui diversas motivações, como a expectativa de contribuição na otimização e eficácia do cuidado ao usuário, melhoria da qualidade e continuidade do tratamento prescrito, bem como a possibilidade de um uso mais eficiente do tempo e dos recursos para os usuários. ${ }^{8}$

A literatura aponta três modelos de prescrição de medicamentos por enfermeiro: prescrição independente, prescrição dependente e grupo protocolo. A prescrição independente é realizada pelo enfermeiro qualificado, responsável por avaliar o indivíduo com uma condição diagnosticada e para tomada de decisões dos cuidados clínicos necessários; neste caso, as prescrições podem ocorrer a partir de uma lista limitada de medicamentos. Na prescrição dependente, o regime de tratamento é determinado pelo prescritor independente (médico), ocorrendo, em seguida, parceria com o prescritor dependente para a implementação do tratamento, as prescrições devem ocorrer a partir de uma lista limitada de medicamentos e em colaboração com o prescritor independente. Por fim, o grupo protocolo consiste em seguir orientações específicas para administrar determinados medicamentos em circunstâncias já planejadas. ${ }^{9}$

Apesar da legalidade da prescrição de medicamentos pela categoria na ESF, percebe-se que não há uma exigência de formação específica para que o enfermeiro exerça adequadamente essa atribuição porque, a maioria das prescrições na atenção primária, enquadram-se no grupo protocolo. ${ }^{9}$ Assim, acredita-se ser indispensável que os currículos de graduação de enfermagem ofereçam embasamento teórico para o melhor preparo para a atuação nesses modelos de prescrição. Todavia, na prática se constata que muitas instituições formadoras não capacitam o acadêmico de enfermagem para 0 exercício dos modelos de prescrição de medicamento.

o cuidado em saúde pela via medicamentosa, associado a outras terapias de cuidado, pode ser uma possibilidade para o enfermeiro, pois é uma característica fundamental para uma abordagem sistêmica do cuidado em saúde. Assim, é possível integrar, consolidar, sistematizar e universalizar políticas de saúde fragmentadas para melhorar a qualidade da assistência. ${ }^{9}$

Esta pesquisa se justifica a partir da apreensão de que a implementação da prescrição/transcrição de medicamentos por enfermeiros no Brasil é permeada por muitos conflitos ético-profissionais, incluindo a necessidade de formação ou o treinamento adequado dos enfermeiros para essa atribuição ${ }^{7}$. Por isso, é pertinente suscitar uma ampla discussão acerca dessa temática entre os enfermeiros. Assim, questiona-se: qual a compreensão dos enfermeiros sobre a prescrição de medicamentos na APS? Dessa forma, este estudo objetiva conhecer a percepção dos enfermeiros sobre a prescrição de medicamentos realizada pela categoria na ESF de um município no Maranhão (MA).

\section{MÉTODO}

Trata-se de um estudo descritivo com abordagem qualitativa, uma vez que esta possibilita maior aproximação com o cotidiano, os motivos e as experiências vividas pelos próprios sujeitos. ${ }^{10} \mathrm{~A}$ metodologia qualitativa detalha as investigações e se preocupa em analisar e interpretar aspectos mais profundos, descrevendo a complexidade do comportamento humano. ${ }^{11}$

O cenário da pesquisa foi a APS do município de Senador La Rocque/MA, localizado no oeste maranhense, com aproximadamente 14.000 habitantes, que dispõe de oito equipes de ESF. Assim, a população do estudo foi composta pelos enfermeiros das equipes, totalizando oito profissionais. Os enfermeiros de cada equipe foram convidados a participar do estudo. Como critério de inclusão, considerou-se o vínculo empregatício como funcionário contratado temporariamente, porque eram esses os profissionais que 


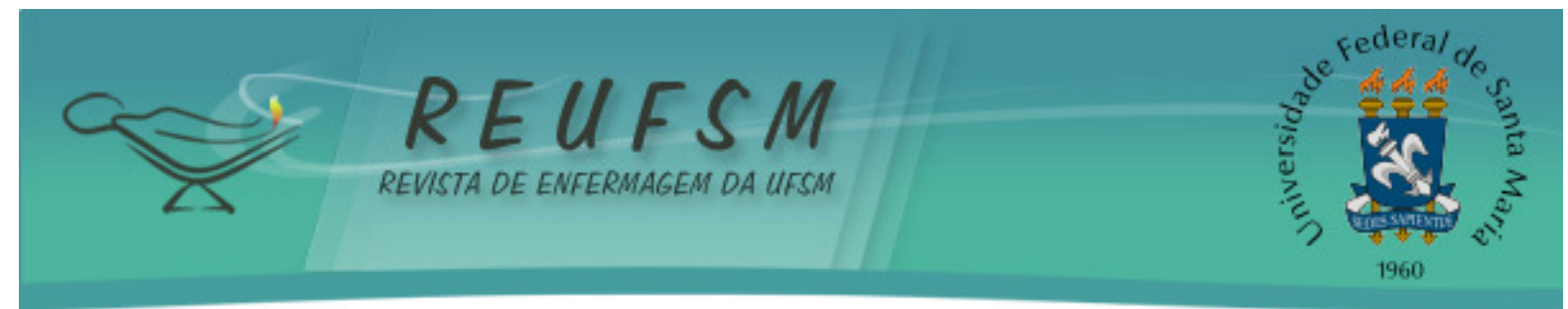

ocupavam o cargo de enfermeiro da ESF do município. Foram excluídos todos os enfermeiros que não responderam a todos os questionamentos da entrevista. A entrevista foi conduzida com os oito enfermeiros das equipes.

Para a coleta de dados, foi utilizada entrevista semiestruturada, elaborada para este estudo, contendo cinco questões que discorriam acerca da percepção sobre a prescrição, as fontes de conhecimento utilizadas para guiar as prescrições, contribuição da formação acadêmica e participação de treinos ou capacitações na área. Os participantes foram abordados individualmente em seu local de trabalho, respeitando as possibilidades de dia e horário, de forma a não prejudicar sua rotina. Antes do início da entrevista, informou-se a cada participante a importância da pesquisa, o objetivo e a utilidade do estudo, assim como lhes foi dada a garantia do anonimato. Todas as entrevistas foram realizadas individualmente, com duração média de 30 minutos e gravadas por meio do aplicativo Gravador de Som \& Voz - ASR para Android no formato "Adaptive Multi-Rate (AMR)".

A participação no estudo foi voluntária, com início após a assinatura do Termo de Consentimento Livre e Esclarecido (TCLE), respeitando os princípios da Resolução do Conselho Nacional de Saúde (CNS) 466/12. O estudo foi aprovado pelo Comitê de Ética/UFMA sob o número 17684713.6.0000.5087. ${ }^{12}$ A coleta de dados foi realizada durante o mês de abril de 2014. Os participantes envolvidos na pesquisa foram identificados por meio de nomes de deuses da mitologia grega, como forma de preservar a sua identidade e manter o anonimato.

Após a coleta dos dados, seguiu-se a conversão das entrevistas que estavam no formato "AMR" para o formato "Moving Picture Experts Group Layer-3 Audio (MP3)" utilizando o software "AMR to MP3 Converter" para que pudessem ser lidas pelo Windows Media Player, facilitando a transcrição.

O tratamento dos dados se fundamentou no método de análise de conteúdo, o qual abrange três fases. ${ }^{13}$ A primeira é a pré-análise, fase da organização propriamente dita, quando se organiza o material a ser explorado com a finalidade de sistematizar as ideias iniciais e torná-las operacionais. A segunda fase consiste na exploração do material, quando se faz a definição das categorias e da codificação, realizando-se a efetivação das decisões tomadas na fase anterior. A terceira fase consiste no tratamento dos resultados obtidos e sua interpretação, em que os resultados brutos são tratados de maneira a serem significativos e válidos. ${ }^{13^{a}}$

Para uma melhor apresentação dos dados coletados neste estudo, inicialmente, foi feita uma caracterização dos profissionais que participaram da pesquisa. Em seguida, aborda-se as categorias estabelecidas a partir de recortes em unidades de registro do texto das entrevistas, a saber: percepção dos enfermeiros sobre a sua prescrição de medicamentos e as bases do conhecimento para a prescrição.

\section{RESULTADOS E DISCUSSÕES}

\section{Caracterização dos participantes}

Todas as entrevistadas eram mulheres que concluíram a graduação em instituições de ensino privado. Quanto ao tempo de atuação na ESF, constatou-se que a maior parte delas, cinco $(62,5 \%)$, trabalhava há menos de um ano. Acredita-se que o curto tempo de atuação é um reflexo da rotatividade de profissionais no município, pois a admissão não é realizada mediante concurso público. Nesse sentido, a instabilidade empregatícia na ESF pode se relacionar à flexibilização do contrato de trabalho e à procura de outras ofertas no mercado com estabilidade contratual e melhores salários. ${ }^{14}$ 


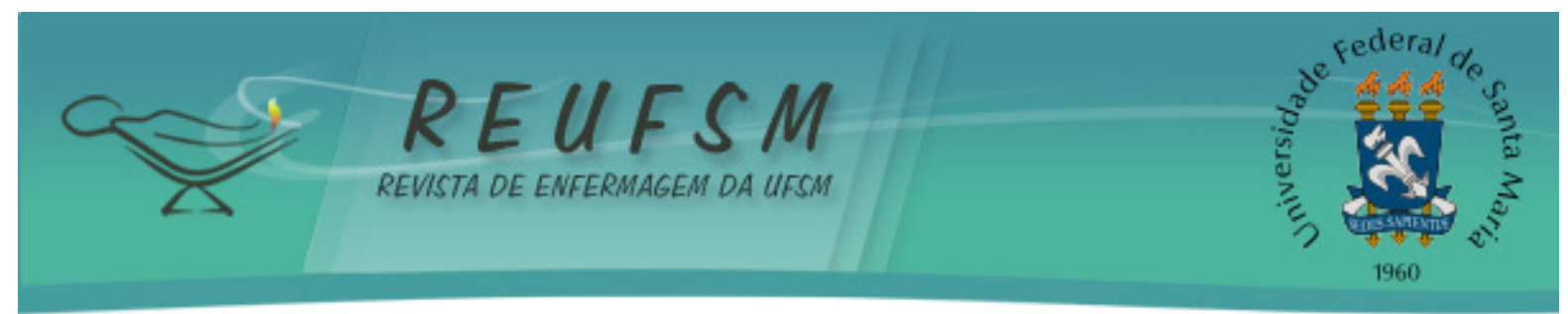

A maioria, sete $(87,5 \%)$, das enfermeiras entrevistadas possui pós-graduação. Os dados deste estudo mostram que a procura e o investimento do enfermeiro em qualificação profissional avançou significativamente. A busca pela qualificação contribui positivamente na prática profissional. Estudos têm demonstrado que a maioria dos profissionais enfermeiros possuem alguma especialização, chegando a $64 \%$ dos enfermeiros da ESF em Vitória, Espírito Santo (ES) e 95\% dos enfermeiros atuantes na ESF de um distrito sanitário de Florianópolis, Santa Catarina. ${ }^{15-17}$

No geral, os enfermeiros das equipes pesquisadas são mulheres jovens, que trabalham no município há pouco tempo, porém apresentam um período de formação superior a três anos com especialização. Das especializações mencionadas, observou-se que sete entrevistadas possuem mais de uma especialidade, sendo Saúde Pública, Saúde da Família, Obstetrícia e Enfermagem do Trabalho as mais cursadas entre as entrevistadas.

\section{Percepção das enfermeiras sobre a prescrição de medicamentos}

Partindo-se da premissa de que a prescrição de medicamentos é um tema permeado por conflitos ético-profissionais, ${ }^{7}$ considerou-se relevante conhecer a opinião das enfermeiras sobre essa atribuição. Assim, ao serem questionadas sobre como veem a prescrição de medicamentos por enfermeiros, as entrevistadas interpretaram essa prática como uma ação positiva e necessária a ser desenvolvida na ESF.

É uma necessidade que eu vejo, pelo menos na minha unidade, é uma forma de ajudar a comunidade. (Deméter)

Eu acho que é necessário, é fundamental nós termos essa liberdade de poder prescrever, porque faz parte da nossa grade, poderia até aumentar um pouquinho mais essa matéria, ser um pouquinho mais específica, mas eu acho fundamental. (Perséfone)

As depoentes consideram que a prescrição de medicamentos pelo enfermeiro é uma prática necessária dentro da APS, uma vez que o profissional de saúde lida com o indivíduo doente, necessitando, muitas vezes utilizar, como intervenção para a reabilitação ou tratamento, a prescrição de medicamentos.

Salienta-se que na prática a reabilitação do usuário nem sempre é medicamentosa. Todavia, o modelo de saúde ainda é predominantemente curativista e hospitalocêntrico. Consequentemente, a terapia medicamentosa tende a ser a resposta que os usuários esperam dos enfermeiros e demais profissionais de saúde. Assim, corre-se o risco de continuar reproduzindo o modelo curativo em detrimento à promoção e prevenção da saúde nas consultas e nos procedimentos de enfermagem. Nesse sentido, espera-se que a categoria considere a prescrição um elemento importante no seu trabalho, mas reconheça que sua função primordial é o cuidado embasado na promoção e prevenção da saúde. ${ }^{18}$

0 enfermeiro possui, dentro dos limites da lei regulamentadora do exercício profissional, respaldo ético-legal para prescrever medicamentos quando previamente estabelecidos em programas de saúde coletiva e em rotina aprovada por instituição de saúde. ${ }^{9}$ Quando o enfermeiro realiza a prescrição de medicamentos com ética e habilidade técnica, contribui para a efetividade das respostas às demandas advindas do serviço de saúde pública e, consequentemente, coopera com a melhoria na qualidade da assistência à população. ${ }^{19}$

Outros relatos dizem respeito à maior permanência do enfermeiro no serviço e à carência por atendimento médico, evidenciando, assim, a importância da prescrição de 


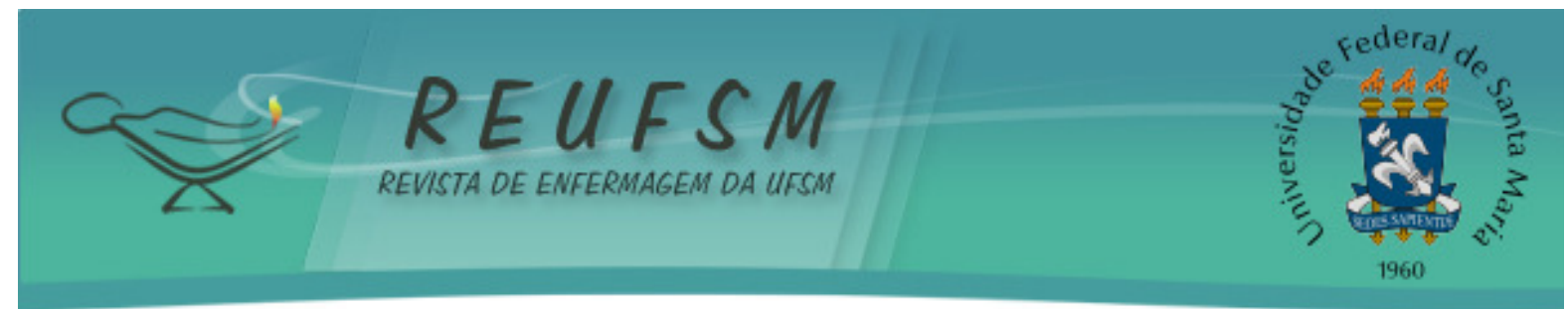

medicamentos realizada por enfermeiros na ESF, conforme pode ser visto nas falas a seguir:

eu acho fundamental, até porque nós passamos a maior parte do tempo no posto de saúde, nós atendemos mais pacientes e nem sempre eles vão querer esperar o médico. (Perséfone)

eu sou a que venho mais na unidade básica do que o médico, eu como enfermeira, então, as pessoas procuram mais a mim. (Anfitrite)

É possível perceber pelos discursos que as enfermeiras veem a prescrição de medicamentos como algo essencial, demonstrando certa satisfação em prescrever. Ao contrário do que demonstrou o estudo realizado no município de Sobral no estado do Ceará, em que os enfermeiros demonstraram insatisfação em prescrever, não somente pelo ato em si, mas por todo o contexto e contradições que vêm ocorrendo nos serviços de saúde do município. ${ }^{3}$

No entanto, a satisfação e a prescrição de medicamentos pelo enfermeiro necessitam de um sólido embasamento que deve advir de fontes variadas, como formação acadêmica, experiência profissional e educação continuada. Como função importante, porém secundária no processo de cuidado da categoria, acredita-se que esse profissional terá uma maior probabilidade de explorar comportamentos e mudanças no estilo de vida dos usuários antes de realizar qualquer prescrição medicamentosa, pois a enfermagem é essencialmente pragmática, característica fundamental para melhorar a aderência dos clientes aos regimes terapêuticos prescritos. ${ }^{18}$

\section{As bases do conhecimento para a prescrição}

Sobre o embasamento para a prescrição de medicamentos, as participantes da pesquisa mencionaram distintas estratégias, tais como: busca na literatura, programas do MS e vivência profissional, conforme referem as citações a seguir:

estudando mesmo muita bula de medicamentos, hoje eu já tenho um pouquinho de experiência, mas antes eu fiz uso de livros para adquirir conhecimento, peguei muita bula e li para aprender, porque é muito complicado, a faculdade disponibiliza, mas é muito por alto; e ajuda de amigos. (Afrodite)

aqui no município, não existe um protocolo de atendimento para questão das prescrições, nós não temos, mas o que acontece, eu costumo prescrever de acordo com a questão dos programas. (Atena)

me baseio muito na minha experiência, na vivência da profissão. (Perséfone)

A partir dos depoimentos se percebeu que esses são métodos utilizados para preencher a necessidade de ofertar uma resposta às exigências de assistência em prescrição de medicamentos aos cadastrados na ESF, constituindo, assim, uma forma de buscar qualificação para exercer adequadamente essa atribuição.,5,7 Para além, observase que a experiência prática é objeto imprescindível para a consolidação do aprendizado. 


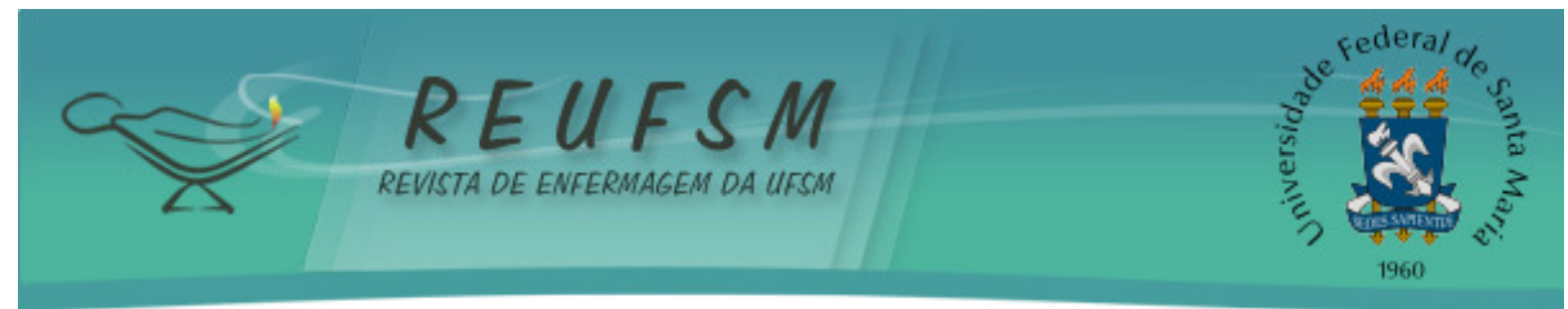

Ainda, torna-se evidente que o município não dispõe de um protocolo de enfermagem que regulamente a prescrição de medicamento, conforme referiu Atena em seu discurso. Assim, protocolos de outros municípios embasam a conduta das profissionais, como pode ser visto a seguir:

o município não dispõe de protocolo, já foi cobrado esse protocolo de medicamento, mas até então eles só alegam que está sendo montado esse protocolo de medicamento, o que nós seguimos é o que o Ministério nos assegura, e outros protocolos de outros municípios vizinhos, que são assegurados pelo Ministério da Saúde. (Demeter)

Os protocolos de atenção à saúde representam um avanço para o enfermeiro na medida em que descrevem as atribuições específicas desse profissional na ESF. Esse tipo de avanço pode ser compreendido como um reconhecimento, pelas políticas públicas, da importância desse profissional na estratégia e na composição da equipe básica de saúde da família, aspecto importante na construção da sua autonomia. ${ }^{20}$

Considerando a construção da autonomia dos enfermeiros, destacam-se os protocolos de enfermagem em Atenção aos Ciclos da Vida utilizados pelos enfermeiros na ESF do município de São Paulo, que representaram uma conquista para a categoria, pois proporcionaram maior autonomia na assistência ao indivíduo e sua comunidade. Porém, faz-se necessário a realização de aperfeiçoamento constante para avaliação e atualização do protocolo e das ações de enfermagem. ${ }^{21}$

Ao serem interrogadas sobre a formação oferecida pelos currículos de graduação para prescrição de medicamentos, as enfermeiras expressaram que, no curso de graduação, as atividades de ensino não foram suficientes para prepará-las devidamente para tal atribuição, como pode ser visto nos relatos a seguir:

olha, assim, eu acredito que não, porque, assim, você sabe como que é a gente sai da faculdade, a gente não sabe muita coisa, mas o que acontece, eu aprendi a prescrever muito com a questão da experiência, porque eu saí da faculdade e eu vim trabalhar aqui, na Estratégia Saúde da Família, assim, eu aprendi com a minha vivência, com a minha prática, a prescrever medicamento, então, a faculdade, ela ensina o básico, é bem superficial, eu só aprendi mesmo com a experiência, trabalhando. (Atena)

seria bom essa grade ser um pouquinho mais específica, ser mais extensa, para que pudéssemos estar realmente preparados. Eu acho que a gente aprende mais na prática. (Perséfone)

$\mathrm{Na}$ fala das entrevistadas, é possível perceber que a instituição formadora ofertou de forma insatisfatória o conhecimento básico necessário para o desempenho dessa atividade. Os dados corroboram com a realidade da formação acadêmica, que ainda apresenta lacunas nesse processo, demandando uma avaliação dos currículos brasileiros para suprir a necessidade de capacitação para a prescrição de medicamentos. ${ }^{22}$

No âmbito internacional, para realizar a prescrição de medicamentos, o enfermeiro deve se submeter a um preparo específico. Na África do Sul, são autorizados pelo Conselho de Enfermagem somente os que estão na saúde pública, após um curso especial e obtenção de licença do Conselho de Farmácia. ${ }^{6}$ Nos EUA, os especializados em práticas avançadas 


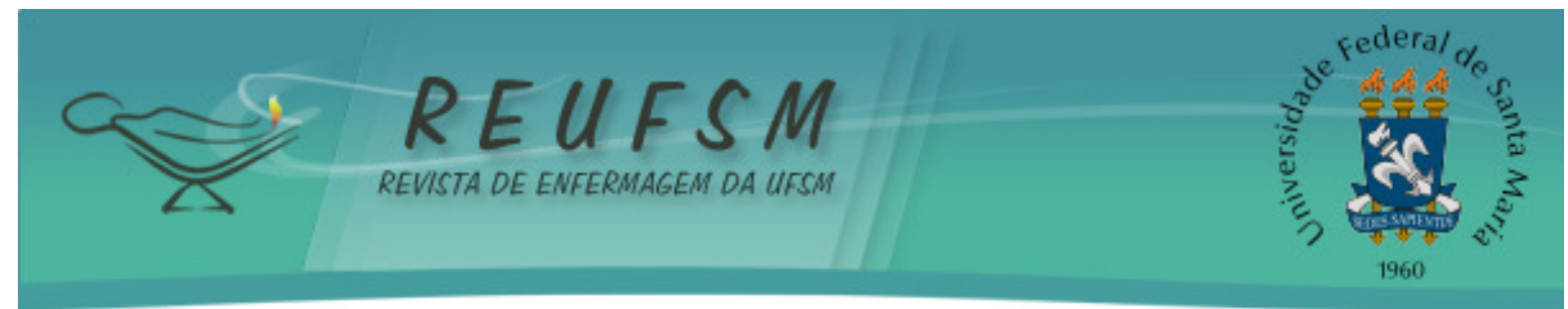

podem receitar em setores de nível primário ou secundário, no entanto, é exigido desses profissionais grau de mestre ou curso especial com estudos sobre diagnósticos e tratamentos de doenças. Os especialistas em clínica, anestesista e obstetrícia também possuem permissão para prescrever fármacos. ${ }^{6}$

Sobre o conhecimento adequado para esta função, as enfermeiras relataram que se sentem qualificadas e preparadas para desenvolvê-la, sendo esse fato resultante da prática profissional das entrevistadas, como pode ser visto nas seguintes declarações:

\section{eu me sinto [...] muito bem capacitada a desenvolver esse trabalho. (Hera)}

mais pela prática, porque a teoria a gente estuda, mas com o tempo você vai pegando aquela segurança. (Hebe)

eu me sinto, pela experiência [...] no início a gente se sente um pouco insegura, não é à toa que a gente precisa da ajuda de uns amigos que já têm experiência até do próprio médico da unidade que também ajuda muito a gente, mas hoje eu já me sinto segura. (Afrodite)

Percebe-se pelas falas que as enfermeiras se sentem capacitadas e seguras para realizar a prescrição, ao contrário do que constatou um estudo em Campina Grande, em que participantes da pesquisa relataram insegurança e despreparo para desenvolver a prescrição. ${ }^{22}$

É importante destacar que algumas das participantes envolvidas nesta pesquisa já realizaram algum tipo de capacitação na área, ou tiveram a oportunidade de serem capacitadas nos cursos de pós-graduação, como pode ser visto nas falas a seguir:

fiz uma graduação em farmacodinâmica. (Hera)

só na especialização, que sou pós-graduanda em saúde pública, a gente também teve uma cadeira de farmacologia. (Artemis)

Embora exista o interesse pela capacitação por parte das enfermeiras envolvidas no estudo, salienta-se a importância da organização e implementação de protocolos de enfermagem, pois eles são fundamentais para nortear as práticas profissionais e padronizar e sistematizar o cuidado, contribuindo para a consolidação do processo de trabalho. ${ }^{23}$

Dos achados neste estudo, destaca-se no relato das enfermeiras de que a formação acadêmica não prepara adequadamente o profissional para a prescrição de medicamentos e que, para preencher essa lacuna, busca-se o conhecimento a partir de cursos de capacitação, leituras individuais sobre os fármacos e acúmulo de experiências diárias. Acredita-se que a necessidade de conhecimento sobre a prescrição de medicamentos só é percebida na prática, quando o profissional é desafiado no serviço a tomar decisões sobre a terapêutica dos usuários. Situação que sinaliza para a importância da readequação do ensino dos cursos de enfermagem no sentido de se aproximarem mais da realidade enfrentada nos serviços de saúde, sobretudo na APS.

A prescrição de medicamentos por enfermeiros foi introduzida na APS por meio dos programas de saúde com o intuito de melhorar a qualidade da assistência e agilizar o atendimento aos usuários. Por isso, a partir dos resultados deste estudo, percebeu-se a necessidade de adequar os programas de graduação e educação continuada de 


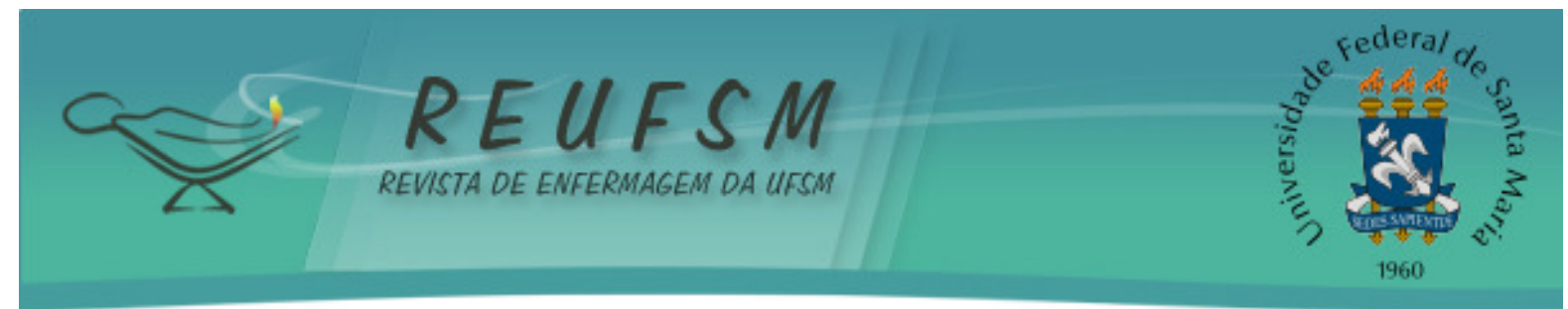

enfermagem à realidade vivenciada nos serviços de saúde, bem como desenvolver a competência do profissional enfermeiro para esse tipo de prescrição.

Notou-se, também, que essa prática para ser efetiva, precisa do apoio organizacional das esferas municipal e federal, pois são nessas instâncias que ocorrem a elaboração dos protocolos locais e a promoção de treinamentos e capacitações contínuas dos profissionais do serviço. Dessa forma, percebe-se que a implementação da prescrição de medicamentos por enfermeiros é uma questão complexa.

Reconhecer a importância da prescrição de medicamentos na categoria não significa desvalorizar a essência da enfermagem que é o cuidado e a prevenção, mas permitir a possibilidade de integrar o cuidado e a cura para prover uma assistência mais sistêmica. Pois, esse profissional continuará priorizando suas habilidades interpessoais no relacionamento com os usuários, enquanto simultaneamente implementam mais habilidades associadas com o modelo médico de cuidado. ${ }^{18}$

\section{CONSIDERAÇÕES FINAIS}

A partir dos discursos se verificou que as enfermeiras visualizam a prescrição medicamentosa como uma atribuição importante e necessária ao município, pelo fato de lidarem com doenças e porque nem sempre o médico está à disposição da comunidade, pois ele é o prescritor independente da ESF no aspecto curativo. As participantes se declararam preparadas e seguras para realizar a prescrição, porém não mencionaram a formação acadêmica como a responsável por essa preparação, e sim a vivência profissional e a formação complementar.

Este estudo mostrou o quão ainda é necessário investir na melhoria das grades curriculares dos cursos de enfermagem, de modo que os enfermeiros sejam instrumentalizados para atuarem em uma realidade complexa, sobretudo na questão da prescrição de medicamentos. A gestão municipal pode contribuir promovendo treinamentos e educação continuada, bem como na implementação de um protocolo local de prescrição de medicamentos na atenção primária.

Destaca-se ainda que o enfermeiro não deve considerar a prescrição de medicamentos como a finalidade do seu processo de trabalho, e sim uma importante complementação da assistência prestada ao usuário, desempenhando dentro da equipe ações de educação em saúde, promoção e prevenção de doenças dentre outras. Assim, esse profissional estará apto a prover uma assistência mais sistêmica aos usuários da APS porque conseguirá integrar o 'cuidado' e a 'cura'.

A limitação do estudo se refere a questão geográfica delimitada. Diante disso, para pesquisas futuras, recomenda-se estender a pesquisa para outros municípios com vistas à composição de um panorama mais ampliado da realidade brasileira.

\section{REFERÊNCIAS}

1. Backes DS, Backes MS, Erdmann AL, Buscher A. O papel profissional do enfermeiro no Sistema Único de Saúde: da saúde comunitária à estratégia de saúde da família. Ciênc Saúde Coletiva [Internet]. 2012 [acesso em 2013 mar 15];17(2):223-30. Disponível em: http: //www.scielo.br/pdf/csc/v17n1/a24v17n1.pdf.

2. Brasil. Ministério da Saúde. Política Nacional de Atenção Básica. Brasília (DF): Ministério da Saúde; 2012.

3. Ximenes Neto FRG, Costa FAM, Chagas MIO, Cunha ICKO. Olhares dos enfermeiros acerca de seu processo de trabalho na prescrição medicamentos na Estratégia Saúde da Família. 


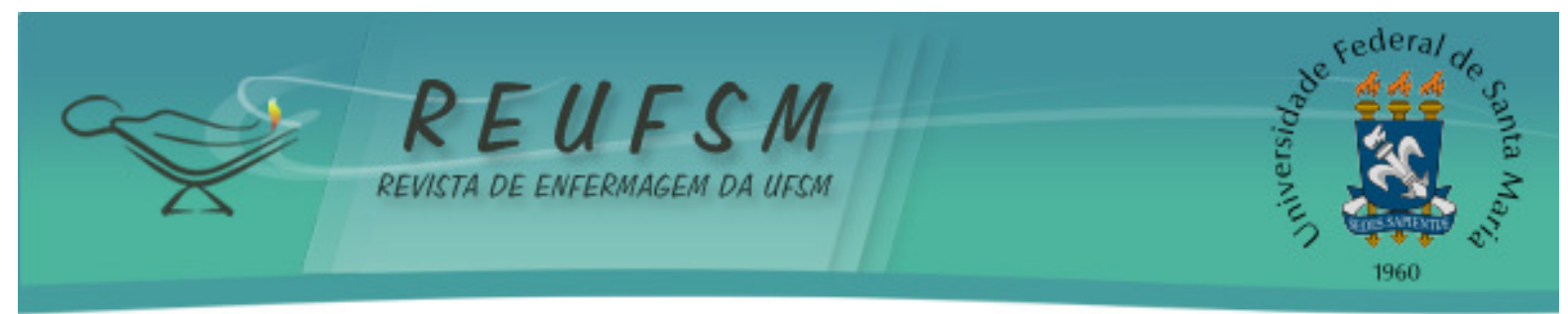

Rev Bras Enferm [Internet]. 2007 [acesso em 2013 mar 12];60(2):133-40. Disponível em: http: //www.scielo.br/pdf/reben/v60n2/a01v60n2.pdf.

4. Brasil. Ministério da Saúde. Portaria $\mathrm{n}^{\circ}$ 2.488, de 21 de outubro de 2011. Aprova a Política Nacional de Atenção Básica, estabelecendo a revisão de diretrizes e normas para a organização da Atenção Básica, para a Estratégia Saúde da Família (ESF) e o Programa de Agentes Comunitários de Saúde (PACS). Diário Oficial da União, Brasília; 2011 out 24. Seção 1, p. 48-55.

5. McBrien B. Personal and professional challenges of nurse prescribing in Ireland. $\mathrm{Br} \mathrm{J}$ Nurs [Internet]. 2015 [acesso em 2015 nov 28];24(10):524-8. Disponível em: http://www.ncbi.nlm.nih.gov/pubmed/26018018.

6. Borges IAL. Consulta de enfermagem, prescrição de medicamentos e solicitação de exames por enfermeiros na atenção básica à saúde. Enferm Foco [Internet]. 2010 [acesso em $2013 \quad$ abr 12];1(1):5-8. Disponível http://revista.portalcofen.gov.br/index.php/enfermagem/article/view/1/1.

7. Kroezen M, Dijk LV, Groenewegen PP, Francke AL. Nurse prescribing of medicines in Western European and Anglo-Saxon countries: a survey on forces, conditions and jurisdictional control. Int J Nurs Stud [Internet]. 2012 [acesso em 2013 maio 21];49(8):1002-12. Disponível em: http://www.ncbi.nlm.nih.gov/pubmed/22369921.

8. Gielen SC, Dekker J, Francke A, Mistiaen P, Kroezen M. The effects of nurse prescribing: a systematic review. Int J Nurs Stud [Internet]. 2014 jul [acesso em 2015 nov 2015];51(7):1048-61. Disponível em: http://www.ncbi.nlm.nih.gov/pubmed/24398118. Doi:10.1016/j.ijnurstu.2013.12.003. Epub 2013 Dec 16.

9. Sodha M, Dhillon S. Non-medical prescribing. London: Pharmaceutical Press; 2009. 241 p.

10. Minayo MCS. O desafio do conhecimento: pesquisa qualitativa em saúde. $14^{a}$ ed. São Paulo: Hucitec; 2014.

11. Lakatos EM, Marconi MA. Metodologia científica. 6a ed. São Paulo: Atlas; 2011.

12. Brasil. Ministério da Saúde. Conselho Nacional de Saúde. Resolução CNS n 466, de 12 de dezembro de 2012. Aprova diretrizes e normas regulamentadoras de pesquisas envolvendo seres humanos. Brasília; 2012.

13. Bardin, L. Análise de conteúdo. Lisboa, Portugal: Edições 70; 2011.

14. Pinto ESG; Menezes RMP; Villa TCS. Situação de trabalho dos profissionais da Estratégia Saúde da Família em Ceará-Mirim. Rev Esc Enferm USP. 2010;44(3):657-64.

15. Silva VG, Motta MCS, Zeitoune RCG. A prática do enfermeiro na Estratégia Saúde da Família: o caso do município de Vitória/ES. Rev Eletrônica Enferm [Internet]. 2010 [acesso em 2016 jan 4];12(3):441-8. Disponível em: http://dx.doi.org/10.5216/ree.v12i3.5278.

16. Silva KM, Santos SMA. A práxis do enfermeiro na Estratégia de Saúde da Família e o cuidado ao idoso. Texto \& Contexto Enferm. 2015;24(1):105-11.

17. Barbosa TSC, Almeida Filho AJ, Santos TCF, Gomes MLB, Oliveira AB, Souza MCF. Políticas de saúde e educação e a oferta dos cursos de especialização em enfermagem: 2001-2007. Rev Enferm UERJ. 2011,19(2):292-8.

18. Bradley E, Nola P. Non-medical prescribing: multidisciplinary perspectives. Cambridge: Cambridge University Press; 2008. 190 p.

19. Monteiro KA, Barbosa SP. Compreensão do Enfermeiro quanto à Prescrição de Medicamentos na Estratégia Saúde da Família. Revista Enfermagem Integrada, Ipatinga 


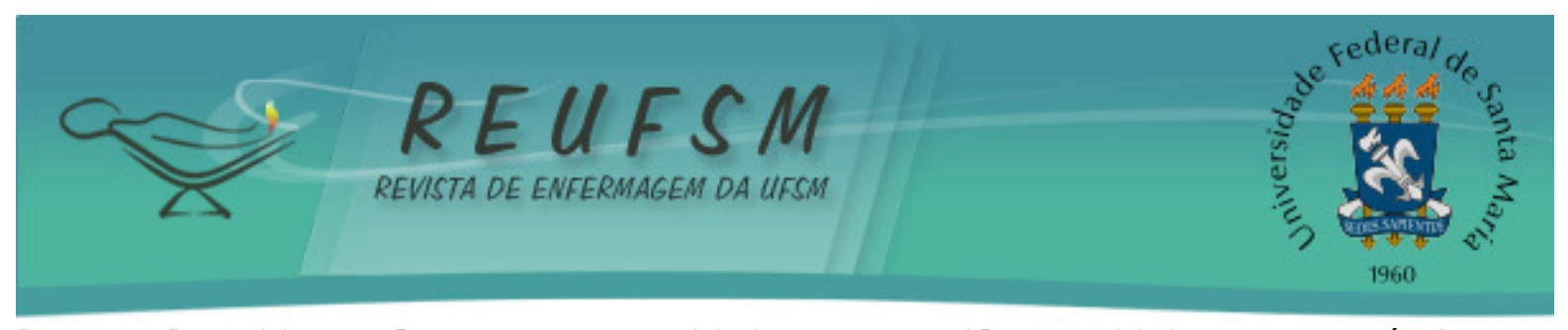

[Internet]. 2011 [acesso em 2013 jun 12];4(1):690-97. Disponível em: http://www.unilestemg.br/enfermagemintegrada/artigo/v4/05-compreensao-doenfermeiro-quanto-a-prescricao-de-medicamentos-na-estrategia-saude-da-familia.pdf.

20. Passos CM. O trabalho do enfermeiro na atenção básica de Belo Horizonte: avaliação das ações programáticas [dissertação]. Belo Horizonte: Universidade Federal de Minas Gerais, Escola de Enfermagem; 2011. 178 p.

21. Ataka T, Oliveira LSS. Utilização dos protocolos de enfermagem no Programa de Saúde da Família no município de São Paulo. Saúde Colet [Internet]. 2007 [acesso em 2013 jun 28];3(13):19-24. Disponível em: http: //www.redalyc.org/pdf/842/84201304.pdf.

22. Vasconcelos RB, Araújo JL. A prescrição de medicamentos pelos enfermeiros na Estratégia Saúde da Família. Cogitare Enferm. 2013 dez;18(4):743-50.

23. Alves KYA, Salvador PTCO, Santos VEP, Martins CCF, Costa TD. Cuidar-curar transpessoal e os protocolos de enfermagem: "cuidado com a vida". Rev Enferm USFM [Internet]. 2014;4(4):858-64. Disponível em: http://cascavel.ufsm.br/revistas/ojs2.2.2/index. php/reufsm/article/view/13188/pdf.

Data de recebimento: $17 / 12 / 2015$

Data de aceite: 23/08/2016

Maria Neyrian de Fátima Fernandes

E-mail: neyrianfernandes@gmail.com

Rua Urbano Santos, SN. Centro.

CEP: 65900-410 - Imperatriz, MA - Brasil 